\title{
Differentiation of pluripotent stem cells to muscle fiber to model Duchenne muscular dystrophy
}

Authors: Jérome Chal ${ }^{1,2,3}$, Masayuki Oginuma ${ }^{1}$, Ziad Al Tanoury ${ }^{1}$, Bénédicte Gobert ${ }^{1}$, Olga Sumara ${ }^{1}$, Aurore Hick ${ }^{4}$, Fanny Bousson ${ }^{4}$, Yasmine Zidouni ${ }^{1}$, Caroline Mursch ${ }^{1}$, Philippe Moncuquet ${ }^{1}$, Olivier Tassy ${ }^{1}$, Stéphane Vincent ${ }^{1}$, Ayako Miyanari ${ }^{1}$, Agata Bera ${ }^{1}$, Jean-Marie Garnier ${ }^{1}$, Getzabel Guevara ${ }^{3}$, Marie Hestin ${ }^{3}$, Leif Kennedy ${ }^{1}$, Shinichiro Hayashi $^{5,6}$, Bernadette Drayton ${ }^{5,6}$, Thomas Cherrier ${ }^{1}$, Barbara Gayraud-Morel ${ }^{7}$,

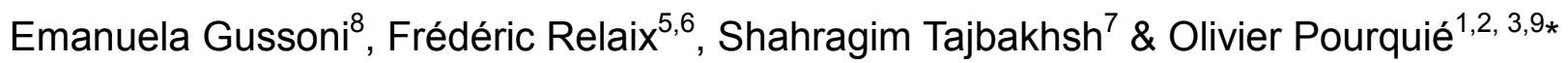

\section{Affiliations:}

${ }^{1}$ Institut de Génétique et de Biologie Moléculaire et Cellulaire (IGBMC), CNRS (UMR 7104), Inserm U964, Université de Strasbourg, Illkirch Graffenstaden. F-67400, France.

${ }^{2}$ Stowers Institute for Medical Research, Kansas City, Missouri 64110, USA.

${ }^{3}$ Department of Genetics, Harvard Medical School and Department of Pathology, Brigham and Women's Hospital, Harvard Stem Cell Institute. 77 Avenue Louis Pasteur, Boston, Massachusetts 02115, USA.

${ }^{4}$ Anagenesis Biotechnologies, Parc d'innovation, 650 Boulevard Gonthier d'Andernach 67400 Illkirch Graffenstaden, France.

${ }^{5}$ UPMC Paris 06, UMR-S 787, F-75013, Paris, France.

INSERM, Avenir team, Pitié-Salpétrière, F-75013, Paris, France.

${ }^{6}$ Institut de Myologie, F-75013, Paris, France.

${ }^{7}$ Institut Pasteur, CNRS URA 2578, Paris, France, 75015.

${ }^{8}$ Division of Genetics and Genomics Boston Children's Hospital, 3 Blackfan Circle, Boston, MA 02115,USA.

${ }^{9}$ Howard Hughes Medical Institute, Kansas City, Missouri 64110, USA. 
${ }^{*}$ Contact Information: Olivier Pourquié, Ph.D.

Department of Genetics, Harvard Medical School and Department of Pathology, Brigham and Women's Hospital, 77 Avenue Louis Pasteur, Boston, Massachusetts 02115

email: pourquie@genetics.med.harvard.edu 


\section{Summary}

Key cell types including skeletal muscle have proven difficult to differentiate in vitro from pluripotent cells. During embryonic development, skeletal muscles arise from somites, which derive from the presomitic mesoderm (PSM). Based on our understanding of PSM development, we established serum-free conditions allowing efficient differentiation of monolayer cultures of mouse embryonic stem (ES) cells into PSM-like cells without introduction of exogenous genetic material or cell sorting. We show that primary and secondary skeletal myogenesis can be recapitulated in vitro from these PSM-like cells. Our strategy allowed for the production of striated contractile fibers from mouse and human pluripotent cells in vitro with an efficiency comparing with current cardiomyocytes differentiation protocols. We also differentiated ES cells into Pax7-positive cells with satellite cell characteristics, including the ability to generate dystrophin-positive fibers when grafted into muscles from dystrophin-deficient $m d x$ mice. We show that differentiated ES cells derived from $m d x$ mice exhibit a striking branched phenotype resembling that described in vivo, thus providing an attractive model to study the origin of the pathological defects associated with Duchenne Muscular Dystrophy.

\section{Introduction}

Skeletal muscles constitute the most abundant tissue in our body. Several important muscle degenerative diseases such as Duchenne Muscular Dystrophy could benefit from cell therapy approaches if healthy muscle progenitors could be grafted in patients to recreate the pools of lost muscles and resident stem cells ${ }^{1}$. The efficient generation of functional muscle cells in vitro from pluripotent cells has so far only been achieved by introducing exogenous DNA into the cells and forcing expression of specific transcription factors such as MyoD, Pax3 or Pax ${ }^{2-7}$. When allowed to differentiate in a variety of conditions, embryoid bodies derived from mouse ES cells can yield a wide range of derivatives from the three germ layers including skeletal muscle ${ }^{8-10}$. However, 
this strategy allows poor control and reproducibility of the differentiation process and usually yields mixed populations with low percentage of the target cell population. Several studies using this approach have recently claimed the production of skeletal muscle from mouse or human pluripotent cells but neither the efficiency of the protocols nor the degree of differentiation of the myogenic cells obtained have been documented 11-16. Alternatively, conditions permitting the differentiation of mouse and human pluripotent cells in monolayers based on the modulation of various signaling pathways to induce a paraxial mesoderm fate have also been reported ${ }^{17-24}$. However, the methods described in most of these studies lead to limited production of paraxial mesoderm precursors necessitating enrichment by cell sorting. Furthermore in all these studies, no evidence for production of contractile millimeter-size fibers with organized myofibrils or of satellite cells in their niche has been provided.

\section{Results}

\section{Analysis of the transcriptome landscape during paraxial mesoderm differentiation}

In order to develop methods allowing efficient differentiation of skeletal myofibers from pluripotent cells, we first set out to recapitulate the early stages of differentiation of the myogenic lineage in the PSM. The posterior to anterior maturation gradient of the PSM means that within a given embryo the different stages of differentiation are spatially mapped out along the posterior-to-anterior axis. This provided the unique opportunity of profiling the full process of differentiation from the tail bud stage to the onset of myogenesis. We generated microarrays from consecutive fragments stretching from the tail bud to the newly formed somite from E9.5 mouse embryos (Fig 1a). This microarray series allowed us to generate a database representing expression of all transcripts expressed in the differentiating PSM (Fig $1 \mathrm{~b}$ and Extended data Fig 1). These histograms provide an accurate representation of gene expression along the PSM when compared with in situ hybridization patterns as exemplified for well characterized genes such as the Fibroblast Growth Factor (FGF) ligand Fgf8 or the retinoic acid biosynthetic enzyme Raldh2, which show anterior or posterior graded expression in the PSM or for Mesp2 which shows a striped expression (Fig 1c). Therefore this microarray series 
provides a faithful representation of the transcriptome regulation during early stages of paraxial mesoderm differentiation.

Using clustering analysis, we further show that the mouse PSM can be subdivided into an anterior and a posterior transcriptional domain (Fig 1d) as previously reported for zebrafish ${ }^{25}$. To identify gene signatures specific for each of these PSM domains, we compared gene expression fold changes between the anterior and posterior domains of the PSM (Fig 1e). This analysis identified 285 genes up-regulated in the anterior PSM and 330 in the posterior PSM with a fold change superior to 2 . These included most genes known to be specific for either posterior or anterior PSM, such as Msgn1 and Pax3 respectively (Fig 1e, Supplementary Table 1).

\section{Wnt activation and BMP inhibition are required for in vitro differentiation of mouse ES cells toward a presomitic mesoderm fate}

We reasoned that a successful myogenic differentiation procedure might need to mimic these important transitions of the developing PSM. To monitor the differentiation of ES cells towards the posterior PSM fate, we generated a transgenic mouse ES cell line (Msgn1-repV for Mesogenin1 reporter Venus) in which the promoter of the PSM posterior-specific gene Msgn ${ }^{26}$ drives expression of the Venus fluorescent protein. Embryos generated by tetraploid aggregation from Msgn1-repV cells faithfully expressed the reporter in the posterior PSM (Fig 2a). To identify secreted factors able to guide differentiation of ES cells toward the posterior PSM fate, we searched for such factors specifically expressed in this domain in the microarray series of differentiating paraxial mesoderm. One such secreted factor showing strong enrichment in the posterior PSM was the Wnt activator R-spondin3 (Rspo3) ${ }^{27}$ (Fig 1e; Supplementary Table S1). By in situ hybridization, Rspo3 exhibits a graded expression specific to the posterior PSM (Fig 2b). When monolayers of Msgn1-repV cells were cultured in serumfree medium supplemented with Rspo3 and the BMP inhibitor, LDN193189 (to block lateral plate mesoderm induction ${ }^{28}$ ), large numbers (45-65\%) of Venus-positive $(\mathrm{M}+)$ cells were observed after 4 days of differentiation (Fig 2c). M+ cells generated in RDL medium did not show significant expression of the lateral plate marker Foxf1a and of the 
cardiac marker $N k \times 2.5$ but showed upregulation of the paraxial mesoderm markers Tbx6, Hes7, and Msgn1 (Fig 2d). At day4, a majority of the M+ cells differentiated in these conditions also expressed the PSM-marker Tbx6 (Fig $2 \mathrm{~g}$ ). We next compared the transcriptional identity of the $\mathrm{M}+$ cells differentiated in vitro to their in vivo counterparts. Microarrays were generated from undifferentiated ES cells and FACS- sorted $\mathrm{M}+$ cells cultured in serum-free RDL medium for 4 days, and we compared the gene expression fold changes between ES cells and $M+$ cells against the fold changes between ES cells and the posterior PSM microarrays described above (Fig 2i, Fig 1d). During differentiation, $\mathrm{M}+$ cells up-regulated a large number of posterior PSM signature genes including Msgn1, Tbx6, Dkk1, Rspo3, Cdx2, and Evx1 as observed in vivo (Fig 2i, Supplementary Table 2). Thus, in vitro differentiation of the Msgn1-repV reporter ES cells in the presence of Rspo3 and a BMP inhibitor efficiently induces their differentiation toward a posterior PSM-like fate. Since Rspo3 is a Wnt activator, we substituted the GSK3 $\beta$ inhibitor CHIRON99021 (Chir) which activates the Wnt pathway to Rspo3 in the differentiation medium (CDL). This led to similar induction of Msgn1+ cells (Fig 2c) which when analyzed using quantitative real time PCR (QRT-PCR) and microarrays showed a very similar profile to the cells differentiated in RDL medium (Fig 2d, Extended data Fig 2).

In vivo, as paraxial mesoderm cells mature, they become located in the anterior PSM, and they down-regulate expression of the posterior PSM markers Msgn1 and Tbx6 while activating expression of $P a x 3$, an early and essential myogenic inducer expressed in the anterior PSM and in myogenic precursors ${ }^{29}$ (Fig 1b, Fig 2a). A similar dynamic was observed in culture in RDL or CDL medium as by day 5 of differentiation $\mathrm{M}+$ cells become Pax3-positive while down-regulating Tbx6, suggesting acquisition of an anterior PSM fate (Fig 2g). To monitor the differentiation of ES cells toward the anterior PSM fate, we generated a mouse ES reporter line (Pax3-GFP) in which the Green Fluorescent Protein (GFP) was placed under the control of Pax3 regulatory sequences. When these reporter cells were cultured in RDL or CDL medium, around 30-50 \% of GFP-positive cells were observed after 6 days of differentiation (Fig 2c). By QRT-PCR, the Pax3-GFP-positive $(\mathrm{P}+)$ cells were found to be essentially negative for the neural marker Sox2 (Fig 2e), but positive for the anterior PSM/somitic markers 
Pax3, Tbx18, Meox2 and Uncx (Fig 2e-f), thus excluding a neural origin for these cells. Expression levels of these genes were comparable to endogenous expression levels in mouse embryonic tails (Fig 2f). Furthermore, these cells express the Pax3 protein (Fig $2 \mathrm{~h}$ ) and exhibit a typical mesenchymal aspect different from the epithelial rosetteforming neural precursors expressing Pax3 observed in neural inducing conditions (Data not shown). We also generated microarrays to compare the transcriptome of $\mathrm{P}+$ cells to that of the anterior PSM. We plotted the gene expression fold changes between ES and $\mathrm{P}+$ cells differentiated in RDL condition media compared to the fold changes between ES and anterior PSM (Fig 2j). During differentiation, ES cells up-regulated a large number of anterior PSM signature genes including Ripply2, Meox2, Pax3, Foxc2, Cer1 and Dmrt2 (Fig 2j, Supplementary Table 3). Very similar differentiation profiles were observed in P+ cells differentiated in CDL medium (Fig 2f, Extended data Fig 2). Thus, in vitro differentiation of the Msgn1-repV and the Pax3-GFP reporter ES cells in the presence of Wnt activators and BMP inhibitor efficiently recapitulates early differentiation stages of the paraxial mesoderm in the PSM. This provided us with a scalable protocol for the generation of large amount of cells that closely resemble the precursors of myogenic cells in vivo.

\section{Recapitulation of primary and secondary myogenesis in vitro leads to production of millimeter-long contractile striated muscle fibers}

We next sought to define conditions in which these anterior PSM-like cells could be reproducibly induced to generate skeletal muscle in vitro. We first differentiated ES cells in RDL or CDL medium for 6 days and then cultured cells in serum-free myogenesis promoting medium containing the myogenesis promoting factors Hepatocyte Growth Factor (HGF), Insulin Growth Factor 1 (IGF-1) and Fibroblast Growth Factor 2 (FGF-2)

${ }^{30}$ in which they were maintained for up to 2 months (Fig 3a). At day 9, differentiating cells have down-regulated Pax 3 and begun to activate Pax7 as is observed for myogenic precursors in vivo ${ }^{29}$. At this stage, many cells have differentiated into proliferating myoblasts (Myod-positive, Fig 3b) and myocytes (Myog-positive, Fig 3b). At day 10 , the Myogenin-positive population accounts for $25-30 \%$ of the cells (Fig $2 \mathrm{c}$ ). To monitor the appearance of committed skeletal muscle cells, we generated a reporter ES 
cell line, Myog-repV, harboring a $1.6 \mathrm{~kb}$ fragment of the Myogenin1 promoter fused to Venus. Venus-positive myocytes harboring a single centrally located nucleus (Fig $3 d$, arrowheads), strongly resembling early myotome cells produced during primary myogenesis, were visible after 1 week in culture in our conditions. The number of cells expressing Pax7, Myod, Myog and fast Myosin Heavy chain (MyHC)-positive cells steadily increased during the second week of differentiation, covering large areas of the culture (Fig 3c). The Myog-repV-positive primary myocytes progressively fused to produce fast MyHC- positive myotubes (Fig 3e), extending up to several hundred of microns by 10 days (Data not shown). Therefore, this suggests that our protocol is able to recapitulate essential steps of primary myogenesis.

After two to three weeks in vitro, a large number of multinucleated Laminin and Dystrophin-positive fibers expressing fast MyHC could be observed in the cultures (Fig 3f-i). After three to four weeks in culture, the more mature (larger) fibers contained about 25-50 myonuclei, a number comparable to that found in perinatal fibers 31 (Supplementary Table 4). We compared by QRT-PCR the expression of a set of myogenic markers in 3-week old cultures differentiated in the conditions described above (Fig 3a) with cultures of undifferentiated ES cells, and with mouse E11.5 trunk muscles (containing primary myofibers) and E17.5 back muscles (in which secondary, foetal myogenesis is ongoing) (Fig 3j). Remarkably, the marker of foetal muscle fibers Nfix ${ }^{32}$ and the Fast2A (Myh2) myosin heavy chain were not observed in E11.5 trunk muscles but were significantly enriched both in the E17.5 muscle sample and in the 25day old cultures (Fig 3j). The muscle fibers differentiated in culture for 3 to 4 weeks reached a density of around 40 fibers per square millimeter, with local fiber patches density reaching 300+ fibers per square millimeter (Supplementary Table 4). Immunolabeling with anti-fast $\mathrm{MyHC}$ antibody showed that these fibers exhibit highly organized striation as expected from mature muscle fibers (Fig $3 \mathrm{k}$ ). Differentiated fibers exhibited spontaneous in vitro contractions, indicating that the sarcomeric organization of the fibers is functional (Data not shown). The dimensions of these fibers were over 1 millimeter in length and around 11 micrometers in width, with a sarcomeric length of about 2.4 micrometers, which compares well with the dimensions of post-natal fibers in vivo ${ }^{31}$ (Supplementary Table 4). Thus, our differentiation protocol recapitulates both 
primary and secondary (foetal) skeletal myogenesis resulting in the production of striated contractile muscle fibers exhibiting a phenotype similar to early post-natal fibers.

\section{Satellite-like cell regenerative properties of the Pax-7 positive cells produced in vitro from mouse ES cells}

In these long-term cultures, fast MyHC-positive fibers were interspersed with a population of small cells expressing Pax7, strongly resembling the satellite precursors cells described in vivo ${ }^{33,34}$ (Fig 3l). After 3-4 weeks in culture, the number of Pax7 positive-cells decreased and the remaining cells were found tightly associated to the myofibers (Fig 3l). Many of the Pax7+ cells were Ki67-negative suggesting that they have exited the cell cycle (Data not shown), and they were sometimes found enclosed in the laminin-positive basal lamina surrounding the muscle fibers as expected for satellite cells (Figure $3 \mathrm{~m}, \mathrm{n}$ ). This data suggest that cells in the mature myogenic cultures spontaneously organize to recreate a satellite cell-like niche in vitro. To test whether the Pax7-positive cells differentiated in vitro exhibit satellite cells properties, we generated a mouse Pax7-GFP transgenic ES reporter line in which GFP expression is driven by a Pax7 BAC transgene. We also introduced an ubiquitous reporter CAGGSCFP in these cells to be able to permanently track their progeny in grafting experiments. We differentiated these cells for 3-4 weeks according to the protocol described above and FACS-sorted the Pax7-GFP positive cells which represented about $0.5 \%$ of the total single cell population. We next grafted $1.10^{5}$ cells into the Tibialis anterior muscle of adult Rag1 $1^{-/}$Dmd ${ }^{\text {mdx }-5 \mathrm{Cv}}$ mice. One month after injection, we observed that GFPexpressing donor cells generated foci of fast MyHC (Fig 4a-b) and Dystrophin-positive fibers (Fig 4c-d). The grafted cells also contributed Pax7-positive satellite-like cells located under the basal lamina of GFP-positive fibers (Fig 4e-g). The donor-derived fibers were well aligned with host fibers, and showed a smaller cross section area compared to host GFP-negative fibers (Fig 4a-d). Therefore, these experiments demonstrate that the Pax7 positive-cells differentiated in vitro can behave as satellite cells when grafted in a Dystrophin-deficient environment. 
Increased branching of dystrophin deficient $m d x$ ES cells differentiated to muscle fibers in vitro

We next derived mouse embryonic stem cell lines from blastocysts of the dystrophin mutant $m d x$. After 3 to 5 weeks of myogenic differentiation in vitro in the serum-free conditions described above (Fig 3a), mature muscle fibers also differentiated in $m d x$ ES cells culture. Differentiated multinucleated $m d x$ fibers reached up to 1.2 millimeters in length with an average diameter of 10.5 micrometers which are values similar to the control fibers. Spontaneously contracting fibers were also observed in differentiated $m d x$ ES cultures (data not shown). Absence of Dystrophin has been suggested to weaken the fiber membrane leading to formation of membrane tears upon contraction, ultimately leading to fiber necrosis ${ }^{35,36}$. To test whether differentiated $m d x$ fibers in vitro exhibit defects in membrane integrity, $m d x$ and control muscle fibers were differentiated for 3-4 weeks in vitro and then stained with Evans blue ${ }^{37}$. Dye uptake was not detected in $m d x$ or in control fibers differentiated in the same conditions whereas it could be observed following membrane permeabilisation with saponin (Extended data Fig 3). Strikingly, in the differentiated $m d x$ ES cultures, around $30 \%$ of the muscle fibers exhibited one or more lateral branches (Fig $5 \mathrm{c}-\mathrm{e}$ ) compared to only about $10 \%$ of branched fibers observed in control culture (Fig $5 a-b$ ). Fibers showing multiple branches resulting in a star-like aspect were essentially restricted to $m d x$ cultures (Fig $5 d$-e). While sarcomeres appeared grossly normal with an average length of 2.4 micrometers, bundling of the myofibrils along the $m d x$ fibers was often disrupted, presenting tilts or twists (Fig $5 \mathrm{c}-\mathrm{d}$ ) resembling those reported in vivo for $m d x$ fibers ${ }^{38}$.

\section{Transposition of the mouse differentiation protocol to human iPS cells allows efficient production of long striated contractile muscle fibers and Pax-7 positive cells}

Finally, we set out to adapt our myogenic differentiation conditions to human iPS cells. We first cultured iPS cells as single cells for 6 days in serum-free medium containing Chir and LDN193189, followed by culture in serum-free medium containing IGF-1, HGF and FGF-2 growth factors (Fig 6a). After 20 days in culture, large fields comprising fast 
MyHC-positive (Fig 6b, e) and Myogenin-positive (Fig 6c) fibers and Pax7-positive cells (Fig 6d) were observed in the differentiating hiPS culture. Between 20 and 30 days of culture, we observed large numbers of MyHC-positive fibers more than one millimeterlong which were intermingled with Pax7-positive cells (Fig 6d-f, data not shown). By 4 weeks, around $22 \%$ of Myog-positive nuclei and 23\% of Pax7-positive nuclei were observed in the cultures. The muscle fibers showed organized sarcomeres as evidenced by the periodic distribution of the sarcomeric proteins Titin and fast MyHC (Fig 6h-j). Furthermore, twitching of these striated fibers was observed in vitro indicating that their sarcomeric organization is functional (Supplementary movie). Strikingly, the diameter of the human muscle fibers generated in vitro was quite smaller than that mouse fibers despite a comparable length (Supplementary Table 4). Many of the Pax7positive cells were also Ki67-negative and were often found lining the MyHC-positive fibers (Fig6 f). The total number of Pax7-positive cells significantly decreased after 40 days down to around $1 \%$ at day 50 . In mouse, around 7 to 10,000 multinucleated muscle fibers could be obtained in wells initially seeded with 20,000 ES cells, whereas in human we obtained around 50-70,000 fibers starting from 75,000 cells, which corresponds to a yield comparing well with current cardiomyocytes differentiation protocols (Supplementary Table 4) ${ }^{39}$. Similar differentiation efficiencies were also observed with the human embryonic stem cells line H9 (data not shown). We compared directly our protocol with that of Shelton et al ${ }^{24}$ in which no BMP inhibitor is added in the first phase of the culture. After 30 days we were unable to detect significant amounts of MyHC- positive fibers or Pax7 cells in iPS cells cultured with these conditions. Significant numbers of small fast MyHC positive myotubes were only detected after 50 days in culture, but they did not show any striation (Extended data Figure 4).

\section{Discussion}

Thus far, difficulties in differentiating pluripotent cells toward a myogenic fate have hampered the development of relevant human in vitro models of muscle diseases such as sarcopenia, cachexia and muscular dystrophies. Here we report the in vitro production from mouse and human pluripotent cells in vitro of striated linear millimeterlong muscle fibers. These fibers can contract and also provide a niche allowing the 
maturation of Pax7-positive satellite-like cells. Such fibers are particularly striking as they do not form in standard myoblast monolayer cultures in which much shorter, irregularly shaped myotubes, lacking striation are usually observed. One reason for the difference between these two types of cultures might lie in the fact that with our protocol, we recapitulate the entire myogenic developmental sequence. In vivo, primary fibers serve as a scaffold for secondary fibers which ultimately organize the linear fusion of myoblastic cells to form adult muscle fibers ${ }^{40}$. In our cultures, we observe formation of primary and secondary muscle fibers suggesting that such a morphogenetic sequence also takes place. These events might also be critical to promote the differentiation of satellite-like cells in vitro. Such self-organization processes arising in ES cell cultures are now widely recognized for a number of structures such as retina, or gut and thus likely occur for muscle tissue as well ${ }^{41,42}$.

We used a series of fluorescent reporter ES cell lines to systematically optimize the differentiation of mouse ES cells toward a paraxial mesoderm fate based on known developmental cues. Transposition of the protocol to human pluripotent cells required several modifications but retained the key elements of the mouse strategy. Notably, human ES cells being equivalent to mouse epiblast cells ${ }^{43}$, the first step of the mouse protocol aiming at inducing epiblast from "naïve" mouse ES cells was unnecessary. Thus, human iPS cells were directly exposed to Wnt activation and to inhibition of the BMP pathway. We found that strong BMP inhibition at this stage is critical, as cells otherwise begin to express BMP4 and drift to a lateral plate fate (data not shown). This step constitutes a significant difference over several protocols reporting paraxial mesoderm induction in which only Wnt signaling is activated ${ }^{14,20,22-24}$. Early addition of FGF-2 was also found to be beneficial to overall survival/proliferation of the human pluripotent cell cultures. We observed that cell cultures induced to a mature PSM fate and exposed to the 3 myogenic growth factors HGF, IGF-1, and FGF-2 ${ }^{30}$ result in a strong induction of myogenesis. While mouse myotubes can be detected between 1 -2 weeks of differentiation, human cultures required about 3 weeks to generate myofibers consistent with the general slower developmental process in differentiating human pluripotent cells compared to mouse ES cells. While virtually nothing is known about the early stages of paraxial mesoderm development and myogenesis in humans, our data 
suggests that signaling cues similar to those described for mouse are deployed during development of the human muscle lineage.

Our work on $\mathrm{mdx}$ ES cells suggests that dystrophin is required for normal morphogenesis of the muscle fibers at least in culture ${ }^{44}$ and provides a novel highly tractable model to study the cell biology of Duchenne Muscular Dystrophy in vitro. Increased branching of the myofibers has previously been reported in vivo in embryos and in adult dystrophic mice, and in mice lacking the olfactory receptor MOR23 or the intermediate filament desmin ${ }^{44-47}$. In adult $m d x$ mice, it has been proposed to result from abnormal fiber morphogenesis caused by defective myoblast fusion arising during the regeneration process stimulated by fibers degeneration $36,45,47$. The increased branching of the $m d x$ fibers differentiated from ES cells in vitro was however unexpected as such a phenotype has not been reported in primary $m d x$ myoblast cultures nor in human DMD myoblasts cultures. The capacity of our cultures to selforganize in these long fibers might explain why such a branching defect is observed. Our in vitro model will allow to explore the origin of the defect in differentiating $m d x$ fibers in detail to analyse whether it is caused by defective fusion of the myogenic precursors.

In mouse, the graft of healthy muscle precursors into $m d x$ recipient has been shown to lead to the production of significant numbers of dystrophin-expressing fibers ${ }^{48}$. Clinical trials in humans using myoblasts amplified in vitro have however failed due to the poor engraftment properties of these cells ${ }^{49}$. To date, mouse studies have established that the cell type presenting the best regenerative properties is the Pax7-positive satellite cell $^{48,50}$. Here we present a new strategy to efficiently produce Pax7-satellite like cells in vitro from pluripotent cells and we show that these cells are able to generate dystrophinpositive muscle fibers and their associated satellite cells when grafted in $m d x$ mice muscle. After further optimization of these differentiation protocols, it may be possible to envision the development of cell therapy strategies for degenerative diseases such as Duchenne muscular dystrophy. 
Acknowledgments: We thank Christopher Henderson, Karim Hnia, Marie Knockaert and members of the Pourquié lab for comments. We are grateful to Jennifer Pace, Tania Knauer-Meyer, Gonçalo Vilhais-Neto, and Merry McLaird for help. We thank Claudine Ebel, Christelle Thibault-Carpentier, Anne Magloth-Roth, and the Imaging Facility. We thank the Microinjection and Phenotyping teams of the Mouse Clinical Institute. We thank Michael Durnin from the Stowers Institute Cell Culture and Animal Facilities. This work was supported by Stowers Institute for Medical Research, by the Howard Hughes Medical Institute, by the FP7 EU grant Plurimes (agreement no. 602423) and by a Strategic grant from the French Muscular Dystrophy Association (AFM) to OP.

Author Contributions J.C. designed and performed experiments, analyzed data and coordinated the project. M.O. performed the PSM microdissection series. Z.A T. transposed the differentiation protocol to hiPS and characterized the human cultures with help from C.M. and B.G..B.G., A.M., G.G. carried out most of the mouse ES differentiation experiments, and M.H carried out hiPS and hES experiments, under J.C. supervision. O.S validated the Myog-repV line. A.H. helped experiments coordination. F.B. contributed to hiPS culture and differentiation. Y.Z. helped develop serum-free protocol. P.M. and O.T. helped with microarray data analysis. T.C. helped analyze $m d x$ cultures. A.B. contributed experimentally to the early project. L.K. provided technical support. J-M. G. generated reporter constructs. S.H., B.D. and F.R. provided the Pax3GFP ES cells. B.G-M. and S.T provided expertise and the Pax7-GFP ES cells. S.V. helped establish $m d x$ ES cells. E.G. provided Rag1-mdx mice and performed transplantation. O.P. conceived and supervised the overall project. O.P. and J.C. performed the final data analysis and wrote the manuscript.

\section{Author Information}

Reprints and permissions information is available at www.nature.com/reprints. The authors declare financial interests: Olivier Pourquié and Jérome Chal are co-founders and share-holders of Anagenesis Biotechnologies, a start-up company specialized in production of muscle cells in vitro for cell therapy and drug screening. Readers are 
welcome to comment on the online version of this article at www.nature.com/nature. Correspondence and requests for materials should be addressed to O.P. (pourquie@genetics.med.harvard.edu). Microarray data were deposited in GEO database under the accession number GSE39615 and pending.

\section{Figure Legends}

\section{Fig 1: Molecular profiling of early stages of paraxial mesoderm differentiation}

(a) Strategy used to produce the microarray series of early paraxial mesoderm differentiation stages. Middle panel shows the left side of the posterior part of an E9.5 mouse embryo indicating the location of the dissected fragments used to generate the microarrays. Tissue fragments were numbered 1 to 7 , along the posterior to anterior axis, starting from the tail bud domain (1), then 5 consecutive fragments of the presomitic mesoderm (PSM) and the forming somite (7).

(b) Diagram illustrating the generation of gene expression histograms from the microarray series. (Left) The 3 series of microdissected PSM fragments used for microarray generation. (Right) Histogram showing Pax3 expression dynamics during PSM differentiation. Red boxes indicate the correspondence between the microdissected samples series, the expression profile histogram (blue bars) and the corresponding transcript expression in the right PSM detected by in situ hybridization (bottom). A single probeset corresponding to Pax3 is shown. The histogram was generated from MAS expression value for a given probeset, with samples of the three microdissection series arranged according to their position along the antero-posterior axis (posterior to the left). The resulting profile gives a quantitative view of transcript expression during early stages of paraxial mesoderm differentiation. TB: Tail bud; S0: last formed somite

(c) Microarray data validation. Comparison of the PSM expression of the genes Raldh2, Fgf8 and Mesp2 detected by in situ hybridization (left) in E8.5 mouse embryos (dorsal view, posterior to the left) with their expression profile in the microarray series; blue 
histograms show the expression levels of a probeset (as described in Fig 1b) corresponding to the genes shown on the left.

(d) Hierarchical clustering of the microarrays of the paraxial mesoderm series. Significant clusters (boxed) identify four major transcriptional domains corresponding to Tail Bud (TB, yellow), posterior PSM (pPSM, green), anterior PSM (aPSM, blue), and Somite (SO, dark blue).

(e) Volcano plot representing gene expression fold changes between anterior and posterior PSM domains vs T-test pValue. Well-characterized signature genes enriched in the posterior PSM (in green) and in the anterior PSM (in blue) are shown. Note that Rspo3 (boxed, in green) is significantly upregulated in the posterior PSM. Total probesets shown is about 45,000 from which about 15,616 are significant $(p<0.05)$.

Fig 2: In vitro differentiation of ES cells toward a Presomitic mesoderm-like fate

(a) (Left) E9.0 mouse embryo hybridized with Msgn1, showing transcript expression restricted to the posterior PSM. (Right) Mouse E9.0 embryos derived from Msgn1-repV ES cells by tetraploid aggregation. In situ hybridization showing that Venus expression is restricted to the posterior PSM (left), while the protein, which is more stable, is detected in the PSM and newly formed somites (right, dorsal view, anterior to the top). Asterisk marks the level of the newly formed somite. TB: tail bud.

(b) Expression of Rspo3 mRNA detected by in situ hybridization in an E9.5 mouse embryo. Asterisk: newly formed somite. Lateral view, anterior to the top.

(c) Induction of Msgn1-repV-positive $(\mathrm{M}+)$, Pax3-GFP-positive $(\mathrm{P}+)$, and Myogenin (MyoG)-RepV -positive cells after respectively 4, 6 and 10 days of differentiation in Rspo3 (RDL) or Chir (CDL) serum free media. Values are expressed as the mean $+/-$ sd.

(d) QRT-PCR analysis of the lateral plate marker Foxf1a, the cardiac marker Nkx2.5 and the PSM markers Msgn1, Tbx6 and Hes7 on FACS-sorted Msgn1-repV -positive $(\mathrm{M}+)$ cells after 4 days in Rspo3 (RDL) or Chir (CDL) serum free media. ES cells expression data is normalized to 1 .Values are expressed as mean $+/$ - sd. 
(e) QRT-PCR analysis comparing the expression level of Pax3, Uncx and Sox2 in undifferentiated ES cells (ESC), and in FACS-sorted Pax3-GFP positive $(\mathrm{P}+)$ and negative $(\mathrm{P}-)$ cells differentiated for 6 days in serum-free RDL medium. $\mathrm{P}$ - expression data is normalized to 1 . Values are expressed as mean $+/$ - sd.

(f) QRT-PCR analysis comparing the expression level of the anterior PSM markers Pax3, Tbx18, Uncx and Meox2 in mouse ES cells, and in FACS-sorted Pax3-GFP positive $(\mathrm{P}+)$ cells after 6 days of differentiation in RDL or CDL medium, and with E9.5 mouse PSM tail. ES cells data are normalized to 1. Values are expressed as mean +/sd.

(g) Comparison of GFP and Tbx6 or Pax3 proteins expression in culture of Msgn1-repV reporter cells differentiated in RDL medium for 4 and 5 days. Scale bar is $400 \mu \mathrm{m}$.

(h) Comparison of GFP and Pax3 proteins in culture of Pax3-GFP reporter cells differentiated in RDL medium for 6 days. Scale bar is $200 \mu \mathrm{m}$.

(i) Plot comparing transcript expression fold changes between ES cells and FACSsorted $\mathrm{M}+$ cells differentiated for 4 days in RDL medium and between ES cells and posterior PSM microarrays. Known signature genes specific for the posterior PSM are highlighted in green. About 3400 significant probesets are shown (fold change with Ttest pValue $<0.05)$.

(j) Plot comparing transcript expression fold changes between ES cells and FACSsorted $\mathrm{P}+$ cells differentiated for 6 days in RDL medium and between ES cells and anterior PSM microarrays. Known signature genes specific for the anterior PSM are highlighted in blue. Around 3400 significant probesets are shown (fold change with Ttest pValue $<0.05$ ).

Fig 3: Differentiation of multinucleated striated muscle fibers and associated Pax7-positive cells from mouse ES cells in vitro 
(a) Schematic representation of the serum free differentiation protocol of mES highlighting key factors applied during the differentiation and the time scale. N: N2 B27, B4: Bmp4, R: Rspo3, D: Dmso, C: Chir, L: LDN193189, H: HGF, I: IGF-1, F: FGF-2, K: KSR. See text for more details.

(b, c) Mouse ES cells cultured for 9 days (b) and 14 days (c) in differentiation medium as shown in (a). Immunohistochemistry with Pax7, MyoD, Myog, Pax3 and Fast Myosin Heavy Chain (MyHC) antibodies. Scale bar is $400 \mu \mathrm{m}$.

(d) GFP live expression in Myog-repV culture differentiated for 9 days in vitro. Scale bar is $100 \mu \mathrm{m}$. Note the mononucleated Venus-positive myocytes (arrowhead)

(e) Myog-repV cells differentiated for 9 days in vitro and stained for GFP (green) and fast MyHC Note the transition stages between the GFP-only expressing myocytes to more mature MyHC expressing cells. Scale bar is $100 \mu \mathrm{m}$.

(f) Mouse ES cells differentiated for 3 weeks in vitro and stained for fast MyHC. Scale bar is $400 \mu \mathrm{m}$.

(g-i) Muscle fibers differentiated in vitro after 3 weeks of culture in serum-free differentiation medium stained for Laminin (g), Dystrophin (h) and fast MyHC (i). Note the continous basal lamina deposit surrounding individual fibers (g), the subsarcolemmal accumulation of Dystrophin (h), and the multinucleated fibers shown DAPI counterstaining (i, arrowheads). Scale bar is $200 \mu \mathrm{m}(\mathrm{g}), 50 \mu \mathrm{m}(\mathrm{h}, \mathrm{i})$.

(j) QRT-PCR analysis comparing expression levels of the myogenic markers Myog, Nfix and Myh2 between ES cells, embryonic E11.5 trunk muscles, foetal E17.5 back muscles and ES cells differentiated for 25 days in serum free- myogenic conditions (D25 LTSF). E17.5 data are normalized to 1. Values are expressed as mean +/- sd.

(k) Higher magnification of muscle fiber differentiated in vitro after 3 weeks of culture in serum-free differentiation medium, stained for Fast MyHC and counterstained with DAPI showing the sarcomeric organization of the fibers. Scale bar is $25 \mu \mathrm{m}$. 
(I) Muscle fibers differentiated in vitro after 3 weeks of culture in differentiation medium, stained for Fast MyHC (red) and Pax7 (green). Note Pax7-positive cells in close proximity to fibers (arrowheads). Scale bar is $100 \mu \mathrm{m}$.

(m) Confocal section showing a high magnification of a muscle fiber differentiated for 24 days in vitro labeled with an anti-laminin (red) and an anti-Pax7 antibody (green), showing a Pax7-positive nucleus (arrowhead) inside the fiber basal lamina (arrowhead). Scale bar is $25 \mu \mathrm{m}$.

(n) Representation of the major developmental stages of ES-derived paraxial mesoderm toward myogenic differentiation. Cellular phenotypes progression and the corresponding reporter genes phases used in this study to monitor the myogenic differentiation are also shown.

\section{Fig 4: Graft of purified Pax7-positive cells differentiated in vitro into adult $\mathbf{m d x}$ muscles}

(a-f) Confocal sections showing immuno-histochemistry on transverse sections of transplanted Tibialis Anterior of 4-months old Rag1 ${ }^{-1-} \mathrm{Dmd}^{\mathrm{mdx}-5 \mathrm{Cv}}$ mice transplanted with FACS-sorted Pax7-GFP -positive cells from 3-weeks (a-d) and 4-weeks (e-g) old myogenic cultures. Grafted mice were analyzed after 1 month (a-d) or 2 months (e-g).

Comparison of Fast MyHC (a) marking the muscle fibers and of GFP expression (labeling the engrafted fibers) (b). The site of injection is assessed by the presence of the 2 micron tracking fluorescent beads $\left(^{*}\right)$.

Comparison of Dystrophin (purple) and Laminin (red) (c) and GFP labeling donorderived fibers (d). All muscle fibers are surrounded by Laminin but only GFP-positive fibers express Dystrophin. Scale bar is $200 \mu \mathrm{m}$.

(e) Pax7-positive nuclei (cyan, arrowhead) can be found under the basal lamina (red). GFP labeling indicating donor origin of the fibers and Pax7+ cell (green) (f). GFP and 
DAPI overlay showing the nuclear localization of GFP in fiber nuclei and in the Pax7positive cell. Scale bar is $50 \mu \mathrm{m}$.

Fig 5: Muscle fibers differentiated in vitro from dystrophin-deficient $\boldsymbol{m d x}$ ES cells show an abnormal branching phenotype

(a-d) Comparison between control $(a, b)$ and $m d x(c, d)$ muscle fibers differentiated in vitro and labeled with an antibody against fast $\mathrm{MyHC}$ showing the branched phenotype (arrowheads) of $m d x$ fibers. (a, c) Scale bar is $500 \mu m$. (b, d) Higher magnification, Scale bar is $100 \mu \mathrm{m}$.

(e) Comparaison of the branching index (in \%) of control and $m d x$ fibers in vitro. Fibers are categorized by number of branches ("b"). Values are represented as mean +/- sd. Statistical significance was evaluated with a Fisher' exact test. ${ }^{* *} p$-value $\leq 0.005$

\section{Fig 6: Myogenic differentiation of human iPS cells}

(a) Schematic representation of the serum free differentiation protocol of hiPS highlighting key factors applied during the differentiation and the time scale. C: Chir, L: LDN193189, F:FGF-2, K: KSR, H: HGF, I: IGF-1. See text for more details.

(b-d) hiPS cells were differentiated as indicated. Cells were fixed after 3 weeks of differentiation and stained for Fast MyHC (b), Myogenin (c) and Pax7 (d). Scale bar $1000 \mu \mathrm{m}$.

(e-g) hiPS cells culture after 40 days of myogenic differentiation, stained for Fast MyHC (e, scale bar is $500 \mu \mathrm{m}$ ), or double-stained for MF20 / Pax7 (f) and Ki67 / Pax7 (g) (Scale bar is $100 \mu \mathrm{m}$ ). 
(h-j) Muscle fibers differentiated in vitro in long-term serum-free cultures and stained for Titin (h) and the fast MyHC (j) (scale bar is $200 \mu \mathrm{m}$ ). (i) High magnification confocal imaging showing the periodic organization of sarcomeres (scale bar is $20 \mu \mathrm{m}$ ).

References

1. Rinaldi, F. \& Perlingeiro, R.C. Stem cells for skeletal muscle regeneration: therapeutic potential and roadblocks. Transl Res (2013).

2. Darabi, R. et al. Human ES- and iPS-derived myogenic progenitors restore DYSTROPHIN and improve contractility upon transplantation in dystrophic mice. Cell Stem Cell 10, 610-619 (2012).

3. Darabi, R. et al. Functional skeletal muscle regeneration from differentiating embryonic stem cells. Nat Med 14, 134-143 (2008).

4. Albini, S. et al. Epigenetic reprogramming of human embryonic stem cells into skeletal muscle cells and generation of contractile myospheres. Cell Rep 3, 661-670 (2013).

5. Salani, S. et al. Generation of skeletal muscle cells from embryonic and induced pluripotent stem cells as an in vitro model and for therapy of muscular dystrophies. J Cell Mol Med 16, 1353-1364 (2012).

6. Tanaka, A. et al. Efficient and reproducible myogenic differentiation from human iPS cells: prospects for modeling Miyoshi Myopathy in vitro. PLoS One 8, e61540 (2013).

7. Abujarour, R. et al. Myogenic differentiation of muscular dystrophy-specific induced pluripotent stem cells for use in drug discovery. Stem Cells Transl Med 3, 149-160 (2014).

8. Braun, T. \& Arnold, H.H. ES-cells carrying two inactivated myf-5 alleles form skeletal muscle cells: activation of an alternative myf-5-independent differentiation pathway. Dev Biol 164, 24-36 (1994).

9. Rohwedel, J. et al. Muscle cell differentiation of embryonic stem cells reflects myogenesis in vivo: developmentally regulated expression of myogenic determination genes and functional expression of ionic currents. Dev Biol 164, 87-101 (1994).

10. Braun, T. \& Arnold, H.H. Myf-5 and myoD genes are activated in distinct mesenchymal stem cells and determine different skeletal muscle cell lineages. EMBO J. 15, 310-318 (1996).

11. Zheng, J.K. et al. Skeletal myogenesis by human embryonic stem cells. Cell Res 16, 713-722 (2006).

12. Mizuno, Y. et al. Generation of skeletal muscle stem/progenitor cells from murine induced pluripotent stem cells. FASEB J 24, 2245-2253 (2010).

13. Tanaka, M. et al. BMP inhibition stimulates WNT-dependent generation of chondrogenic mesoderm from embryonic stem cells. Stem Cell Res 3, 126141 (2009). 
14. Hwang, Y. et al. Directed in vitro myogenesis of human embryonic stem cells and their in vivo engraftment. PLoS One 8, e72023 (2013).

15. Chang, $H$. et al. Generation of transplantable, functional satellite-like cells from mouse embryonic stem cells. FASEB J 23, 1907-1919 (2009).

16. $\mathrm{Xu}, \mathrm{C}$. et al. A zebrafish embryo culture system defines factors that promote vertebrate myogenesis across species. Cell 155, 909-921 (2013).

17. Sakurai, H. et al. In vitro modeling of paraxial mesodermal progenitors derived from induced pluripotent stem cells. PLoS One 7, e47078 (2012).

18. Sakurai, $\mathrm{H}$. et al. Bidirectional induction toward paraxial mesodermal derivatives from mouse ES cells in chemically defined medium. Stem Cell Res 3, 157-169 (2009).

19. Sakurai, H., Okawa, Y., Inami, Y., Nishio, N. \& Isobe, K. Paraxial mesodermal progenitors derived from mouse embryonic stem cells contribute to muscle regeneration via differentiation into muscle satellite cells. Stem Cells 26, 1865-1873 (2008).

20. Borchin, B., Chen, J. \& Barberi, T. Derivation and FACS-Mediated Purification of PAX3+/PAX7+ Skeletal Muscle Precursors from Human Pluripotent Stem Cells. Stem Cell Reports 1, 620-631 (2013).

21. Hosoyama, T., McGivern, J.V., Van Dyke, J.M., Ebert, A.D. \& Suzuki, M. Derivation of myogenic progenitors directly from human pluripotent stem cells using a sphere-based culture. Stem Cells Transl Med 3, 564-574 (2014).

22. Gouti, M. et al. In vitro generation of neuromesodermal progenitors reveals distinct roles for wnt signalling in the specification of spinal cord and paraxial mesoderm identity. PLoS Biol 12, e1001937 (2014).

23. Tan, J.Y., Sriram, G., Rufaihah, A.J., Neoh, K.G. \& Cao, T. Efficient derivation of lateral plate and paraxial mesoderm subtypes from human embryonic stem cells through GSKi-mediated differentiation. Stem Cells Dev 22, 18931906 (2014).

24. Shelton, M. et al. Derivation and expansion of PAX7-positive muscle progenitors from human and mouse embryonic stem cells. Stem Cell Reports 3, 516-529 (2014).

25. Ozbudak, E.M., Tassy, O. \& Pourquie, O. Spatiotemporal compartmentalization of key physiological processes during muscle precursor differentiation. Proc Natl Acad Sci U S A (2010).

26. Wittler, L. et al. Expression of Msgn1 in the presomitic mesoderm is controlled by synergism of WNT signalling and Tbx6. EMBO Rep 8, 784-789 (2007).

27. Kazanskaya, O. et al. R-Spondin2 is a secreted activator of Wnt/betacatenin signaling and is required for Xenopus myogenesis. Dev Cell 7, 525534 (2004).

28. Tonegawa, A., Funayama, N., Ueno, N. \& Takahashi, Y. Mesodermal subdivision along the mediolateral axis in chicken controlled by different concentrations of BMP-4. Development 124, 1975-1984 (1997).

29. Hutcheson, D.A., Zhao, J., Merrell, A., Haldar, M. \& Kardon, G. Embryonic and fetal limb myogenic cells are derived from developmentally distinct 
progenitors and have different requirements for beta-catenin. Genes Dev 23, 997-1013 (2009).

30. Charge, S.B. \& Rudnicki, M.A. Cellular and molecular regulation of muscle regeneration. Physiol Rev 84, 209-238 (2004).

31. White, R.B., Bierinx, A.S., Gnocchi, V.F. \& Zammit, P.S. Dynamics of muscle fibre growth during postnatal mouse development. BMC Dev Biol 10, 21 (2010).

32. Messina, G. et al. Nfix regulates fetal-specific transcription in developing skeletal muscle. Cell 140, 554-566 (2010).

33. Relaix, F., Rocancourt, D., Mansouri, A. \& Buckingham, M. A Pax3/Pax7dependent population of skeletal muscle progenitor cells. Nature 435, 948953 (2005).

34. Sambasivan, R. \& Tajbakhsh, S. Skeletal muscle stem cell birth and properties. Semin Cell Dev Biol 18, 870-882 (2007).

35. Rahimov, F. \& Kunkel, L.M. The cell biology of disease: cellular and molecular mechanisms underlying muscular dystrophy. J Cell Biol 201, 499-510 (2013).

36. Allen, D.G. \& Whitehead, N.P. Duchenne muscular dystrophy--what causes the increased membrane permeability in skeletal muscle? Int $J$ Biochem Cell Biol 43, 290-294 (2010).

37. Matsuda, R., Nishikawa, A. \& Tanaka, H. Visualization of dystrophic muscle fibers in $\mathrm{mdx}$ mouse by vital staining with Evans blue: evidence of apoptosis in dystrophin-deficient muscle. J Biochem 118, 959-964 (1995).

38. Friedrich, $\mathbf{O}$. et al. Microarchitecture is severely compromised but motor protein function is preserved in dystrophic mdx skeletal muscle. Biophys $J$ 98, 606-616 (2010).

39. Minami, I. et al. A small molecule that promotes cardiac differentiation of human pluripotent stem cells under defined, cytokine- and xeno-free conditions. Cell Rep 2, 1448-1460 (2012).

40. Biressi, S., Molinaro, M. \& Cossu, G. Cellular heterogeneity during vertebrate skeletal muscle development. Dev Biol 308, 281-293 (2007).

41. Eiraku, M. et al. Self-organizing optic-cup morphogenesis in threedimensional culture. Nature 472, 51-56 (2011).

42. McCracken, K.W. et al. Modelling human development and disease in pluripotent stem-cell-derived gastric organoids. Nature 516, 400-404 (2014).

43. Li, W. \& Ding, S. Human pluripotent stem cells: decoding the naive state. Sci Transl Med 3, 76ps10 (2011).

44. Merrick, D., Stadler, L.K., Larner, D. \& Smith, J. Muscular dystrophy begins early in embryonic development deriving from stem cell loss and disrupted skeletal muscle formation. Dis Model Mech 2, 374-388 (2009).

45. Chan, S. \& Head, S.I. The role of branched fibres in the pathogenesis of Duchenne muscular dystrophy. Exp Physiol 96, 564-571 (2011).

46. Goodall, M.H., Ward, C.W., Pratt, S.J., Bloch, R.J. \& Lovering, R.M. Structural and functional evaluation of branched myofibers lacking intermediate filaments. Am J Physiol Cell Physiol 303, C224-232 (2012). 
47. Pavlath, G.K. A new function for odorant receptors: MOR23 is necessary for normal tissue repair in skeletal muscle. Cell Adh Migr 4, 502-506 (2010).

48. Montarras, D. et al. Direct isolation of satellite cells for skeletal muscle regeneration. Science 309, 2064-2067 (2005).

49. Mouly, V. et al. Myoblast transfer therapy: is there any light at the end of the tunnel? Acta Myol 24, 128-133 (2005).

50. Collins, C.A. et al. Stem cell function, self-renewal, and behavioral heterogeneity of cells from the adult muscle satellite cell niche. Cell 122, 289-301 (2005).

\section{Online Methods}

\section{Generation of mouse reporter and $m d x$ ES cell line}

Msgn1-repV line. The Msgn1-repV transgene consists of a $6.5 \mathrm{~kb}$ fragment of the mouse Mesogenin 1 promoter ${ }^{26}$ inserted upstream of the Venus (YFP) coding sequence ${ }^{51}$ followed by the SV40 poly-adenylation sequence and a neomycin resistance cassette flanked by FRT sites. Mouse ES cells (E14, 129P2 genetic background, BayGenomics) were transfected with the linearized construct using Lipofectamin (Invitrogen). Clones were selected for Neomycin resistance and validated by the tetraploid aggregation technique as described ${ }^{52}$. One clone, called Msgn1-repV, showing the appropriate expression pattern was selected for further studies.

Pax3-GFP line. Pax3GFP ES cells were generated by homologous recombination using a targeting construct derived from that described in ${ }^{53}$. Briefly, the Pax3GFP(Gene $X$-IRESnLacZ) allele contains $2.4 \mathrm{~kb}$ of the 5' genomic region of Pax3, but lacks the coding sequence of exon 1 , and $4 \mathrm{~kb}$ of the 3 ' sequence which contains exons $2-4$. The insert construct is composed of the coding sequence for GFP, followed by a Puromycin selection cassette flanked by FRT sites. The targeting vector was electroporated into CK 35 ES cells (129Sv genetic background) ${ }^{54}$. ES cells were selected and screened for recombination events by Southern blot analysis using EcoRV digestion and a 5'-flanking probe. The positive clones were further verified by using 3 ' external and internal probes. 
Targeted ES cells were recovered with a $0.5 \%$ frequency, and efficient germline transmission was used to assay cells' pluripotency. In order to generate Pax3GFP ES cells, targeted ES cells were electroporated with a construct containing the FLP recombinase.

Myog-repV line. The same strategy as for Msgn1-repV was used with the Myog-repV transgene using a $1.6 \mathrm{~kb}$ fragment of the mouse Myogenin promoter ${ }^{55}$. One clone, called Myog-repV, showed colocalisation of the fluorescent reporter with the endogenous Myogenin protein in muscle fibers differentiated from embryoid bodies as described ${ }^{9}$ and was selected for further studies.

Pax7-GFP blue line. Pax7-GFP mES clones were derived from the transgenic mice Tg: Pax7-nGFP described in ${ }^{56}$. Cells were cultured in medium containing $1 \mu \mathrm{M}$

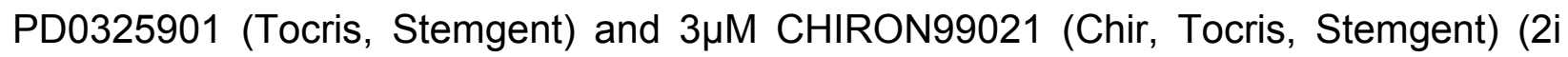
medium) as described ${ }^{57}$. Cells were transfected with an ubiquitous reporter contruct composed of CAGG-nlsCFP followed by the SV40 poly-adenylation sequence and a puromycin resistance cassette flanked by FRT sites. Picked clones were isolated and further amplified by passages in $2 \mathrm{i}$ medium. Two clones were further selected for myogenic differentiation and grafting experiments. The Pax7-GFP label is necessary to sort the cells to be grafted and the CFP label is required to track the descendants of these cells in the host. Thus muscle fibers deriving from the grafted Pax7+ cells are expected to express only CFP whereas satellite cells derived from the grafted cells are expected to express both GFP and CFP. While GFP and CFP expression is expected to be nuclear we have observed leakage of the fluorescent proteins to the sarcoplasm of muscle fibers in which both cytoplasmic and nuclear staining was observed.

Mdx ES cells. $m d x$ mES clones were derived from the dystrophin mutant $m d x$ homozygous/hemizygous blastocysts of the C57BL/10 genetic background (Jackson laboratory) cultured in $2 \mathrm{i}$ medium as described ${ }^{57}$. Ten clones were isolated by picking and further amplified by passages in $2 \mathrm{i}$ medium. Two clones (male, hemizygous) were further selected for the myogenic differentiation study. Labeling of the muscle fibers derived from the $m d x$ ES cells with an anti-dystrophin antibody confirmed the absence 
of the protein (data not shown).

\section{Mouse ES Cell culture and differentiation}

Maintenance. Undifferentiated mouse ES cells were cultured on feeders of mitomycin$\mathrm{C}$ inactivated mouse embryonic fibroblasts at $37^{\circ} \mathrm{C}$ in $5 \% \mathrm{CO} 2$, in a maintenance medium composed of DMEM supplemented with $15 \%$ fetal bovine serum (Millipore), penicillin, streptomycin, $2 \mathrm{mM}$ L-Glutamine, $0.1 \mathrm{mM}$ non essential amino acids, $0.1 \% \beta$ mercaptoethanol $, 1,500 \mathrm{U} / \mathrm{mL}$ LIF and $2 \mathrm{i}$ inhibitors ${ }^{57}$. Cells were passaged by trypsinization (Invitrogen). Prior to differentiation, cells were passaged once onto gelatin-coated, feeder-free culture plates.

Serum-free differentiation of mouse ES cells toward a PSM-like fate. ES cells were trypsinized and plated at various densities in gelatin-coated, feeder-free, 96, 24 and 6well plates directly in serum-free N2B27 medium supplemented with $1 \%$ Knock-out Serum Replacement (KSR, Gibco), 0.1\% Bovine serum albumin (Gibco) and BMP4 at $10 \mathrm{ng} / \mathrm{mL}$ (Peprotech) for 2 days. Cells were then changed to a DMEM-based medium, with $15 \% \mathrm{KSR}$, supplemented with recombinant $10 \mathrm{ng} / \mathrm{mL}$ Rspo3 (Peprotech, R\&D

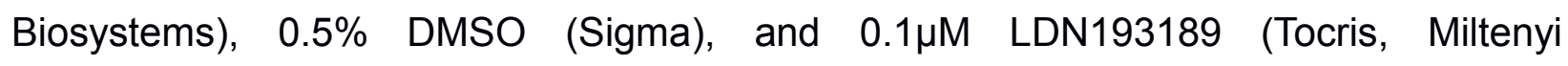
Biotec/Stemgent) (RDL medium) for 4 days. Alternatively, Rspo3 was replaced with CHIRON99021 (Chir; Tocris, Stemgent) at $1 \mu \mathrm{M}$. Differentiation experiments were performed at least 20 times independently.

Serum-free Myogenic differentiation of the mouse PSM-like cells After the 4 days of culture in RDL or CDL medium, cells were changed to a DMEM-based medium with $15 \% \mathrm{KSR}, 0.1 \%$ BSA supplemented with 10ng/ml HGF, $2 \mathrm{ng} / \mathrm{ml}$ IGF-1, 20ng/ml FGF-2

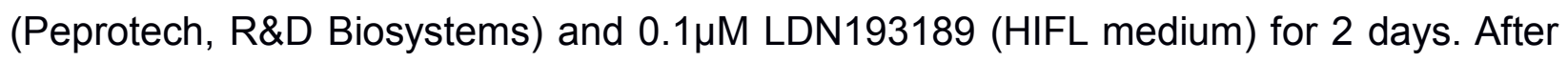
day 8 of differentiation, only HGF, IGF-1, FGF-2 were supplemented (HIF medium). Media was changed every other day. Long-term differentiation experiments were performed at least 15 times independently, on more than 5 different mouse ES cell lines. 


\section{Human PS Cell culture and differentiation}

Maintenance. Undifferentiated human hiPS $11 \mathrm{a}^{58}$ and $\mathrm{H}^{59}$ : hES cells were cultured on Matrigel (BD Biosciences)- coated dishes in mTeSR1media (Stemcell Technologies). Cells were passaged as aggregates.

Serum-free Myogenic differentiation of the human iPS cells. hPS colonies were dissociated with Accutase (Stemcell Technologies) and plated as single cells per well on Matrigel -coated 24 and 12-well plates (approximately 15-18,000 cells $/ \mathrm{cm}^{2}$ ) in $\mathrm{mTeSR} 1$ supplemented with ROCK inhibitor (Y-27632, Sigma) for one day. The medium was changed to a DMEM-based medium supplemented with Insulin-Transferrin-Selenium

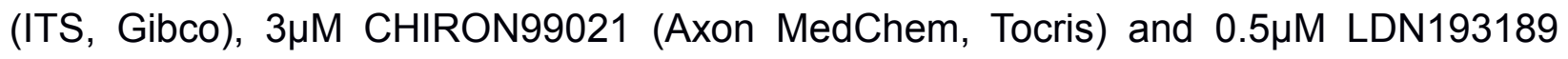
(Miltenyi Biotec/Stemgent) (CL medium). At day 3, 20ng/ml FGF-2 (R\&D Systems) was added for additional 3 days. After 6 days of differentiation, cells were changed to a DMEM-based medium supplemented with 10ng/ml HGF, 2 ng/ml IGF-1, 20ng/ml FGF-

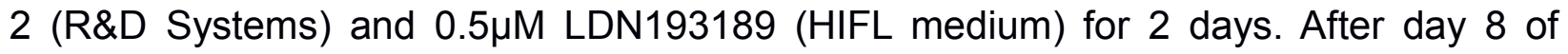
differentiation, cells were cultured in DMEM, $15 \% \mathrm{KSR}$, supplemented with $2 \mathrm{ng} / \mathrm{ml}$ IGF1 for 4 days, and then supplemented with both $10 \mathrm{ng} / \mathrm{ml}$ HGF and $2 \mathrm{ng} / \mathrm{ml} \mathrm{IGF-1}$ after day12. Media was changed every day until day12, and every second day thereafter. Long-term differentiation experiments were performed at least 15 times independently, on 2 unrelated hiPS and one hES lines. All human iPS and ES experiments were done according to local regulations (IGBMC and Brigham and Women's Hospital) and in agreement with national and international guidelines.

\section{Flow cytometry analysis and FACS}

Analysis. Cells were dissociated by trypsinization and analyzed by flow cytometry on a FACS calibur (BD Biosciences) according to GFP expression. Gating was determined for each reporter line using corresponding undifferentiated culture as a baseline control. Data are represented as \% of GFP-positive cells in the culture.

Sorting. Msgn1-repV+ $(\mathrm{M}+)$ and Pax3GFP+ $(\mathrm{P}+)$ cells were isolated by FACS from Msgn1-repV and Pax3-GFP cells respectively differentiated for 4 days and 6 days in $\mathrm{RDL}$ (or $\mathrm{CDL}$ ) medium. Gated fractions were sorted either on FACS Aria (BD 
Biosciences) or S3 cell sorter (Biorad). Pax7-GFP+ cells were isolated from the Pax7GFP blue ES line differentiated for 3-4 weeks in serum-free medium. Data were further analyzed with the FlowJo software. Sorted populations were either processed for microarray analysis, QRT-PCR or for transplantation experiments.

\section{Quantitative RT-PCR}

Total RNA was extracted from ES cell cultures using Trizol (Invitrogen) or with the RNeasy micro-kit (Qiagen). RT-PCR was performed on 5ng total RNA using QuantiFast SYBR Green RT-PCR Kit (Qiagen) and gene specific primers (Primerbank) and run on a LightCycler 480II (Roche). Beta-Actin was used as an internal control. For mouse tail/PSM reference, mouse E9.5 tails were microdissected posterior to the level of the forming somite (SO) and pooled for total RNA extraction. For embryonic and foetal muscle references, mouse E11.5 trunk muscles and mouse E17.5 back muscles were microdissected for total RNA extraction.

\section{Microarrays generation and analysis}

Generation of the PSM microarray series. Microdissections of CD1 mouse E9.5 embryos were done essentially as described ${ }^{60}$. Caudo-rostral series of consecutive $\sim 100 \mu \mathrm{m}$ fragments were collected. 3 series of 7 fragments were generated, from the tailbud to the newly formed somite (S0) level (Fig 1a-b). RNA from each fragment was extracted with Trizol (Invitrogen) and used to generate probes hybridized on GeneChip Mouse Genome 4302.0 arrays.

Microarrays. Biotinylated cRNA targets were prepared from total RNA using a double amplification protocol according to the GeneChip ${ }^{\circledR}$ Expression Analysis Technical Manual: Two-Cycle Target Labeling Assay (P/N 701021 Rev.5, Affymetrix, Santa Clara, USA). Following fragmentation, cRNAs were hybridized for 16 hours at $450 \mathrm{C}$ on GeneChip Mouse Genome 4302.0 arrays. Each microarray was then washed and stained on a GeneChip fluidics station 450 and further scanned with a GeneChip Scanner 3000 7G. Finally, raw data (.CEL Intensity files) were extracted from the scanned images using the Affymetrix GeneChip Command Console (AGCC) version 3.1 . 
Microarray data analysis. Initial filtering and preprocessing, including background correction, quantile normalization and summarization, was performed using both RMA and MAS with the $\mathrm{R}$ Bioconductor package ( $\mathrm{R}$ version 2.12.1, Bioconductor version 2.8). Expression sets were then filtered according to Calls information. Initial filtering and preprocessing, was performed with the $R$ Bioconductor package ( $R$ version 2.12.1, Bioconductor version 2.8). Gene expression fold changes and their corresponding T-test Pvalues between conditions/samples were analyzed using the "Comparative Marker Selection" module of GenePattern ${ }^{61}$. Volcano and FcFc data plots were generated using the Multiplot application of GenePattern. Hierarchical clustering was performed on Microarray RMA data with Bootstrap $p$ values, Euclidean distances and Average-based cluster method. Histogram expression profiles of gene probesets were generated from MAS values. Further analysis was performed using the Manteia database ${ }^{62}$.

\section{Cell preparation and transplantation into Tibialis Anterior muscles.}

Serum-free cultures of 3-4 weeks old differentiated Pax7-GFP blue mouse ES cell were pretreated with ROCK inhibitor (Y-27632, Tocris), one day before trypsin dissociation. Cell preparation was filtered through $30 \mu \mathrm{m}$ mesh (Partec) and GFP-positive cells were FACS-sorted. Target cells were resuspended in PBS with tracking fluorescent beads (Molecular probes). $20 \mu \mathrm{l}$ of cell preparation containing 80,000 to 100,000 cells were injected in the Tibialis Anterior (TA) muscles of 3 to 4-months old Rag1 ${ }^{-/-} \mathrm{Dmd}^{\mathrm{mdx}}{ }_{-5 \mathrm{Cv}}$ male mice $(n=5)$. Injections were done under general anesthesia. Grafted TA muscles were collected 1 to 2 months after transplantation and processed for cryosection and immunohistochemistry. Experiments on mice were done according to local regulations (Boston Children's Hospital, Brigham and Women's Hospital, Institut Pasteur, IGBMC), in agreement with national and international guidelines.

\section{In situ hybridization and immunohistochemistry.}

Whole mount in situ hybridization was carried out as described ${ }^{63}$. Probes for mouse Msgn1, Rspo3, and Pax3 were synthesized from mouse embryo cDNA. Probe for Venus was synthesized from the Venus plasmid (A kind gift of $\operatorname{Dr} A$ Miyawaki) and probe 
for Mesp $2{ }^{64}$, Raldh2 ${ }^{65}$ and Fgf8 ${ }^{66}$ were already described. Images were acquired on a Leica M125 stereomicroscope.

For Immunohistochemistry, cell cultures were fixed for 2 hours in $4 \%$ formaldehyde. Cultures were rinsed three times in PBS, followed by Blocking buffer composed of Trisbuffered saline (TBS) supplemented with $1 \%$ FBS and $0.1 \%$ Triton-X100. Primary antibodies were then diluted in blocking buffer and incubated overnight at $4^{\circ} \mathrm{C}$. Cultures were then washed 3 times with TBST (TBS supplemented with $0.5 \%$ Tween-20) and incubated with secondary antibodies (1:500) and Dapi (5 microgram $/ \mathrm{ml}$ ) in blocking buffer for 2 hours. Cultures were ultimately washed with TBST followed by PBS, before analysis. Dissected TA muscles were prepared for cryosections $(12 \mu \mathrm{m})$ as described in 67. Tissue sections were incubated overnight with primary antibodies in blocking buffer. Secondary antibodies conjugated with AlexaFluor (Molecular probes) were used at 1:500 in Blocking buffer.

Antibodies used in this study are anti-Tbx6 (a kind gift of $\operatorname{Dr} Y$ Saga ${ }^{68}$ ), anti-Dystrophin (Mandra1, Sigma), anti-Dystrophin (Dys1, Leica/Novocastra), anti-Myogenin (Dako, DHSB), anti-Pax3 (DSHB), anti-Pax7 (DSHB), anti-MyoD (Dako), anti-Laminin (Abcam), anti-Titin (DHSB), anti-GFP (Abcam), anti-Fast MyHC (MY-32, Sigma), antiMyHC (MF20, DSHB) and anti-Ki67 (Abcam).

\section{Membrane permeability assays.}

Stage-matched 3 to 4 week-old control and $m d x$ ES were cultured as follows. After 4-6 days of culture in RDL medium, cells were changed to a medium supplemented with the FGF inhibitor PD173074 (Sigma) at $250 \mathrm{nM}, 0.5 \%$ DMSO, and LDN193189 at $0.1 \mathrm{mM}$. After 2 days of culture in this medium, cells were transferred to a myogenic differentiation medium containing $4 \mathrm{mM}$ L-Glutamine, $0.1 \mathrm{mM}$ non essential amino acids and $2 \%$ Horse serum (PAA) in DMEM, in which they were maintained for up to 5 weeks. This protocol results in the same myogenic differentiation efficiency as the serum-free protocol described above but leads to less overall cell death resulting in better Evans blue dye staining. Muscle fibers cultures were treated with Evans Blue dye $(0.2 \%$, Sigma) in N2B27 medium at $37^{\circ} \mathrm{C}$ for 45 minutes. Extra dye was washed away with PBS and cells were reincubated further for 30 minutes in culture medium, prior to 
observation. As a permeabilisation control, cultures were preincubated with Saponin (Sigma) at $50 \mu \mathrm{g} / \mathrm{ml}$ in culture medium at $4^{\circ} \mathrm{C}$ for 20 min prior to staining with Evans Blue. Cells were washed with PBS, and cold culture medium was added prior to observation.

\section{Quantifications and statistics}

\section{Myofibers density count}

A fiber density estimate was calculated by counting the number of fibers in 3 to 4 weeks old control cultures. Quantifications were manually done on an average of 5-10 pictures (at 40-100x magnification) per well, acquired from MyHC-stained myofibers fields. Alternatively, staining was quantified by performing automated cell count using the Array scan XTI (Thermofisher) with a 10x objective (144 fields/well). Image analysis was performed with HCS Studio software. Using the Tube Formation Bioapplication we identified fibers according to Myosin staining and measured their area, length and width. Three independent differentiation experiments were quantified. Results are expressed as averages of number of fibers per square millimeter.

\section{Myofibers branching quantification}

A branching index was calculated by scoring the number of branches per fiber in 3 to 5 weeks old control or $m d x$ cultures ( 2 cell lines per genotype). Quantifications were done on an average of 5-10 pictures sets (at 200X magnification) per well, acquired from $\mathrm{MyHC}$ and Laminin-stained myofibers fields. Five independent differentiation experiments were scored for branching. A total of $600+$ fibers per genotype were scored. Result are expressed in \% of fibers. Statistical analysis was performed using Fisher's exact test. Differences between groups were considered significant for $p$ values $\leq 0.05$.

Myogenic marker quantification. For each marker, immunolabelling was quantified manually on 3 myogenic fields per well. Alternatively, staining was quantified by performing automated cell count using the Array scan XTI (Thermofisher). Nuclei were segmented using DAPI staining and after a threshold was applied on FITC channel intensity to identify Marker-positive cells. 
Experiment was performed in triplicate. Result is expressed as a \% of positive nuclei.

\section{Image acquisition and Processing}

Live or fixed brightfield and fluorescent images were acquired either on a Zeiss Axiovert, Evos FL, Zeiss LSM780, or Leica SP5 systems. Images were processed with Adobe Photoshop, and quantification and measurements were done with Fiji ${ }^{69}$ software. For muscle contractions, movies were taken with a Leica DMRB microscope using a photometric FX camera and a $20 x$ objective. Images were taken at $100 \mathrm{~ms}$ intervals. For movie processing, image sequences were imported and processed in Fiji. Movies were saved in $\mathrm{AVI}$ format at a frequency matching the acquisition rate of 33 frames per second. 


\section{Extended data Figures}

Extended data Fig 1: Generation of a database of gene expression profiles in the differentiating PSM.

Top: Histograms representing expression profiles (generated as described in Fig 1b) of genes associated to major signaling pathways involved in PSM patterning and differentiation. Bottom: schematic representation of the signaling gradients and of the major posterior paraxial mesoderm domains (color-coded) identified by clustering analysis (Fig 1d). Orange bar marks the determination front level where cells acquire their segmental identity.

Extended data Fig 2: Microarrays Gene signature expression analysis in $M+$ and $P+$ differentiated in CDL medium.

(a) Plot comparing transcript expression fold changes between ES cells and FACSsorted $\mathrm{M}+$ cells differentiated for 4 days in CDL medium and between ES cells and posterior PSM microarrays. Known signature genes specific for the posterior PSM are highlighted in green. About 3400 significant probesets are shown (fold change with Ttest pValue $<0.05$ ).

(b) Plot comparing transcript expression fold changes between ES cells and FACSsorted P+ cells differentiated for 6 days in CDL medium and between ES cells and anterior PSM microarrays. Known signature genes specific for the anterior PSM are highlighted in blue. Around 3400 significant probesets are shown (fold change with Ttest pValue <0.05).

(c) Plot comparing transcript expression fold changes between FACS-sorted $\mathrm{P}+$ and $\mathrm{M}+$ cells differentiated CDL medium and between ES cells and $\mathrm{P}+$ and $\mathrm{M}+$ cells differentiated RDL medium. Known signature genes specific for the posterior PSM (green) and anterior PSM (blue) are highlighted. Around 3400 significant probesets are shown (fold change with T-test pValue $<0.05$ ).

Extended data Fig 3: Membrane integrity of $\boldsymbol{m d x}$ muscle fibers differentiated in vitro. Control $(a, b)$ and $m d x$ myofibers $(c, d)$ cultures stained with Evans Blue dye 
(EBD) with and without prior membrane permeabilization with saponin. Scale bar 200 $\mu \mathrm{m}$

Extended data Fig 4: Comparison of differentiation efficiency with the Shelton et al, (2014) protocol.

hiPS cells were differentiated either according the protocol described in this study, (Chal et al, 2015) or according to Shelton et al, 2014.; for 4 weeks (a) and 6-7 weeks (b) respectively.

(a) Side by side comparison of 4 -weeks old cultures stained for Fast MyHC (upper panels), and MyHC (MF20, red) /Pax7 (green) (lower panels). Cell distributions (DAPI staining, blue) are shown for each field as an upper right corner inset. Scale bar $500 \mu \mathrm{m}$. (b) 6-7 weeks old culture stained for Fast MyHC, at lower (upper panels, counterstained with DAPI) and higher (lower panels) magnifications. Scale bars are 400 and $100 \mu \mathrm{m}$, respectively. 


\section{Supplementary information}

\section{Supplementary tables}

Supplementary Table 1: Lists of signature genes differentially expressed between posterior and anterior PSM. Genes showing a fold change superior to 2 and an associated p-Value inferior to 0.05 are shown. Msgn1 which shows a fold change of 1.9 has been added to the list. Expression of the genes highlighted in yellow in the posterior or anterior PSM domains has been validated based on literature, on MGI (Mouse Genomics Institute) expression data or by in situ hybridization (data not shown). Together, these genes define signatures for the posterior and anterior PSM respectively.

Supplementary Table 2: List of signature genes of the posterior PSM upregulated in differentiated Msgn1-repV positive cells in vitro. Posterior PSM signature genes from Supplementary Table 1 which are also up-regulated in the differentiated Msgn1repV positive cells. Expression of the genes highlighted in yellow has been validated based on literature, on MGI expression data or by in situ hybridization (data not shown).

Supplementary Table 3: List of signature genes of the anterior PSM upregulated in differentiated Pax3-GFP positive cells in vitro. Anterior PSM signature genes from Supplementary Table which are also up-regulated in the differentiated Pax3-GFP positive cells. Expression of the genes highlighted in yellow has been validated based on literature, on $\mathrm{MGI}$ expression data or by in situ hybridization (data not shown).

\section{Supplementary movies}

Supplementary Movie: Contractions of human muscle fibers differentiated from iPS cells in vitro. 4 weeks-old culture. 
Online References

51. Nagai, T. et al. A variant of yellow fluorescent protein with fast and efficient maturation for cell-biological applications. Nat Biotechnol 20, 87-90 (2002).

52. Nagy, A., Rossant, J., Nagy, R., Abramow-Newerly, W. \& Roder, J.C. Derivation of completely cell culture-derived mice from early-passage embryonic stem cells. Proc Natl Acad Sci U S A 90, 8424-8428 (1993).

53. Relaix, F. et al. The transcriptional activator PAX3-FKHR rescues the defects of Pax3 mutant mice but induces a myogenic gain-of-function phenotype with ligand-independent activation of Met signaling in vivo. Genes Dev 17, 2950-2965 (2003).

54. Kress, C., Vandormael-Pournin, S., Baldacci, P., Cohen-Tannoudji, M. \& Babinet, C. Nonpermissiveness for mouse embryonic stem (ES) cell derivation circumvented by a single backcross to 129/Sv strain: establishment of ES cell lines bearing the Omd conditional lethal mutation. Mamm Genome 9, 998-1001 (1998).

55. Cheng, T.C., Wallace, M.C., Merlie, J.P. \& Olson, E.N. Separable regulatory elements governing myogenin transcription in mouse embryogenesis. Science 261, 215-218 (1993).

56. Sambasivan, R. et al. Distinct regulatory cascades govern extraocular and pharyngeal arch muscle progenitor cell fates. Dev Cell 16, 810-821 (2009).

57. Ying, Q.L. et al. The ground state of embryonic stem cell self-renewal. Nature 453, 519-523 (2008).

58. Boulting, G.L. et al. A functionally characterized test set of human induced pluripotent stem cells. Nat Biotechnol 29, 279-286 (2011).

59. Thomson, J.A. et al. Embryonic stem cell lines derived from human blastocysts. Science 282, 1145-1147 (1998).

60. Dequeant, M.L. et al. A complex oscillating network of signaling genes underlies the mouse segmentation clock. Science 314, 1595-1598 (2006).

61. Reich, M. et al. GenePattern 2.0. Nat Genet 38, 500-501 (2006).

62. Tassy, O. \& Pourquie, O. Manteia, a predictive data mining system for vertebrate genes and its applications to human genetic diseases. Nucleic Acids Res (2013).

63. Henrique, D. et al. Expression of a Delta homologue in prospective neurons in the chick. Nature 375, 787-790 (1995).

64. Saga, Y., Hata, N., Koseki, H. \& Taketo, M.M. Mesp2: a novel mouse gene expressed in the presegmented mesoderm and essential for segmentation initiation. Genes Dev 11, 1827-1839 (1997).

65. Niederreither, K., Subbarayan, V., Dolle, P. \& Chambon, P. Embryonic retinoic acid synthesis is essential for early mouse post-implantation development. Nat Genet 21, 444-448 (1999).

66. Crossley, P.H. \& Martin, G.R. The mouse Fgf8 gene encodes a family of polypeptides and is expressed in regions that direct outgrowth and patterning in the developing embryo. Development 121, 439-451 (1995). 
67. Mathew, S.J. et al. Connective tissue fibroblasts and Tcf4 regulate myogenesis. Development 138, 371-384 (2011).

68. Yasuhiko, Y. et al. Tbx6-mediated Notch signaling controls somite-specific Mesp2 expression. Proc Natl Acad Sci U S A 103, 3651-3656 (2006).

69. Schindelin, J. et al. Fiji: an open-source platform for biological-image analysis. Nat Methods 9, 676-682 (2012). 
a

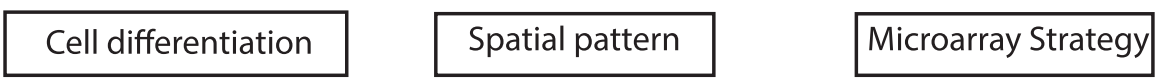

Myotome Sclerotome
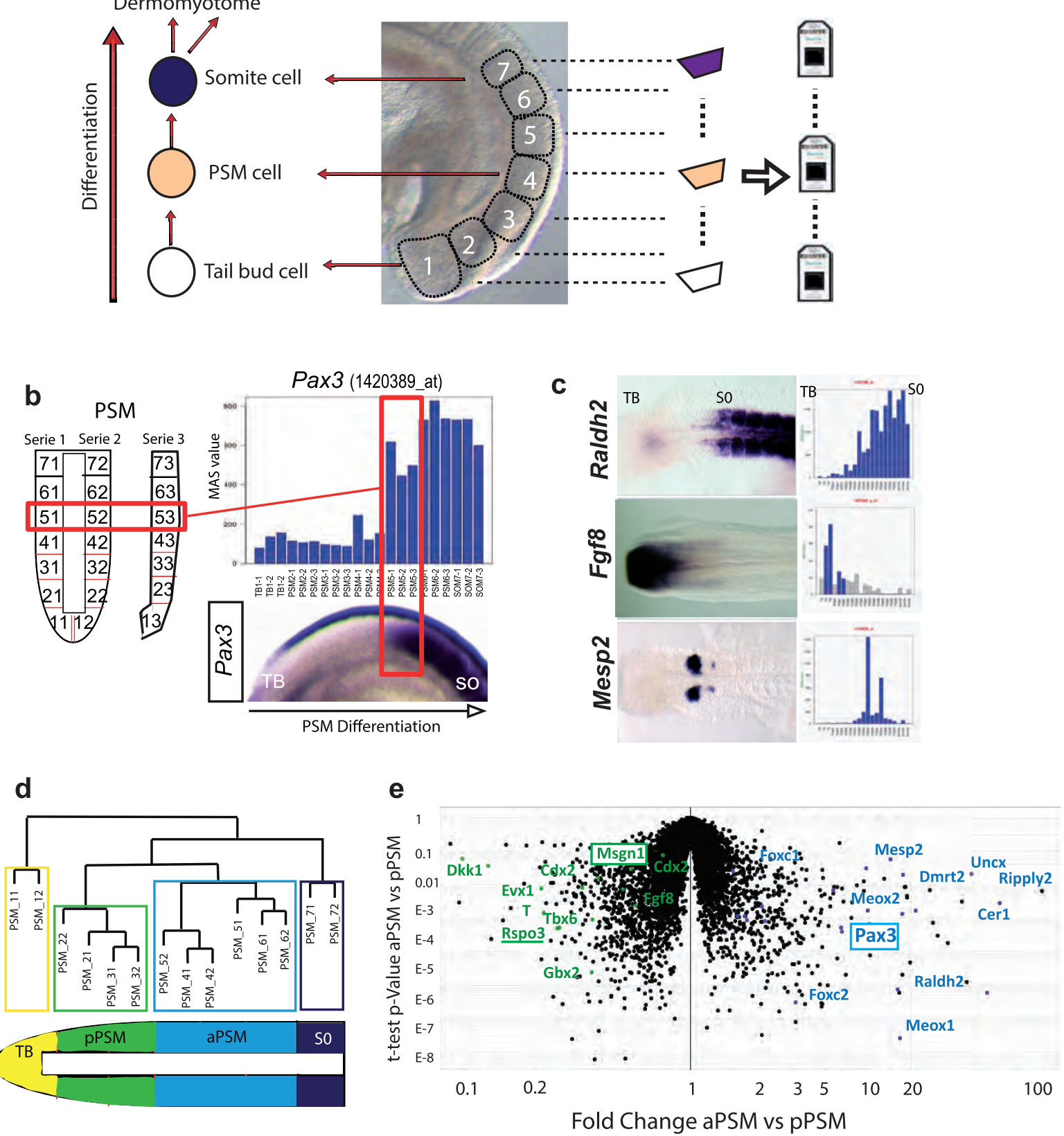

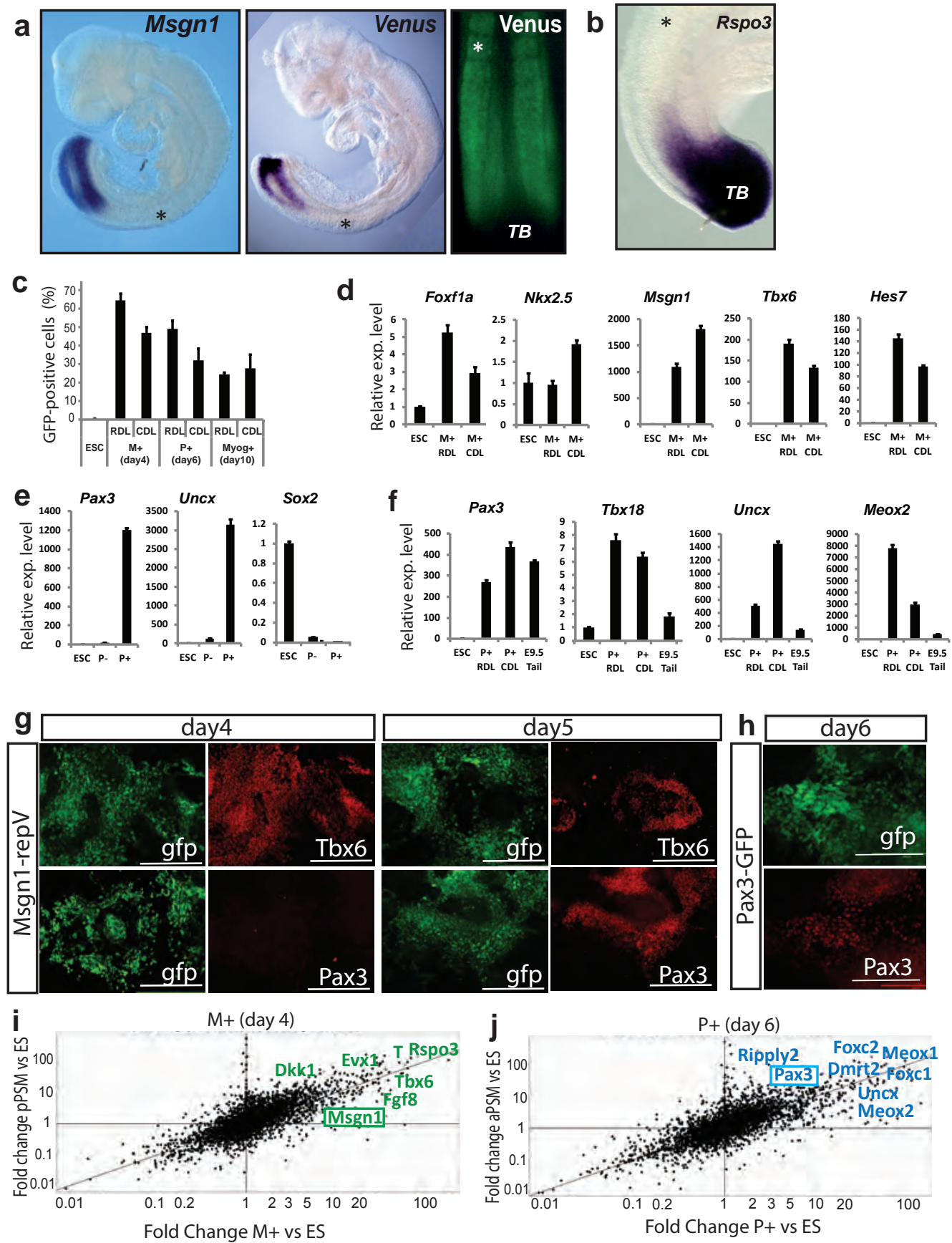
a

\begin{tabular}{c|ccc}
\multirow{2}{*}{ mES } & $0-2 \mathrm{~d}$ & $2-4 \mathrm{~d}$ & $6-8 \mathrm{~d}$ \\
\cline { 3 - 3 } & NK-B4 & K-RDL (CDL) & K-HIFL
\end{tabular}
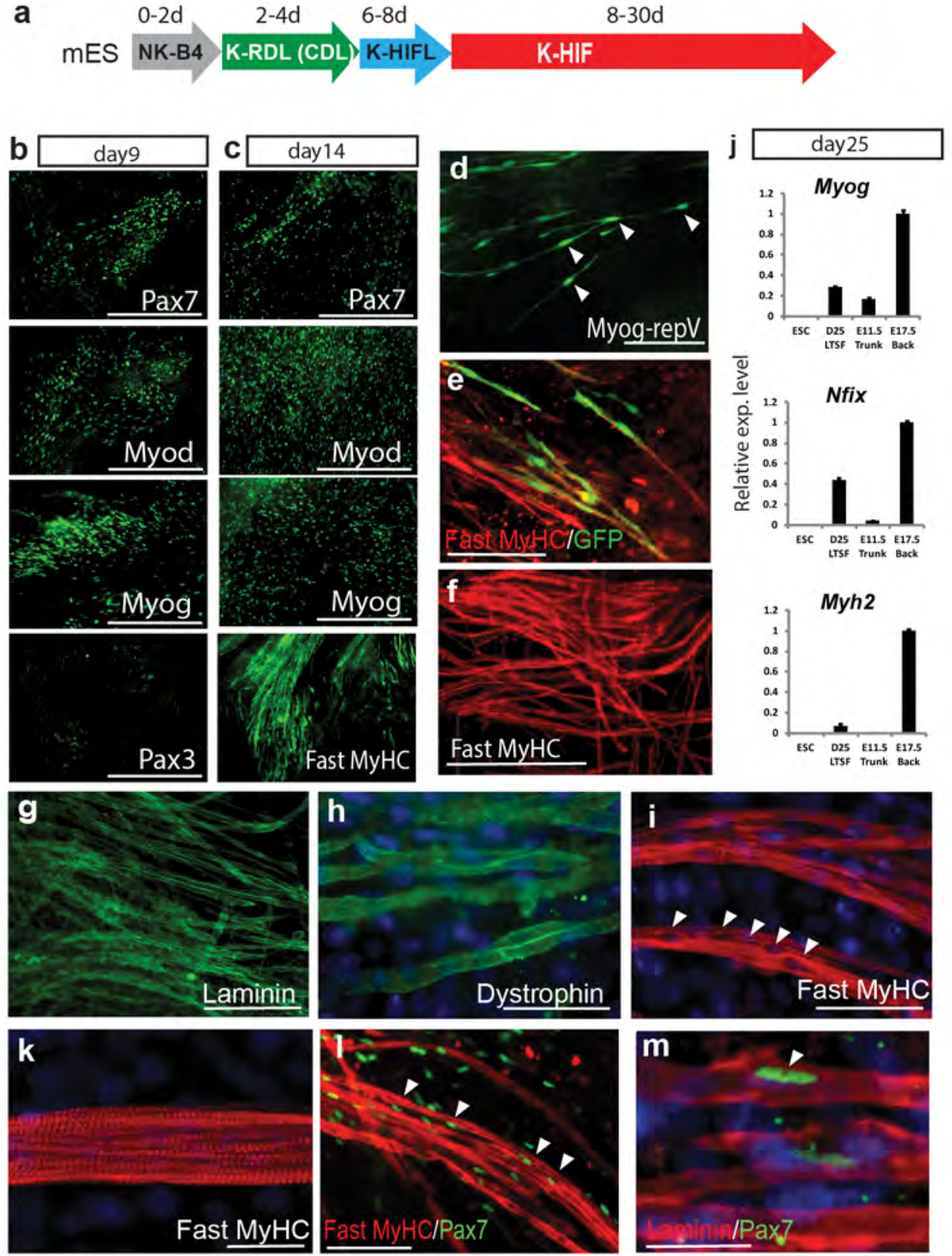

n

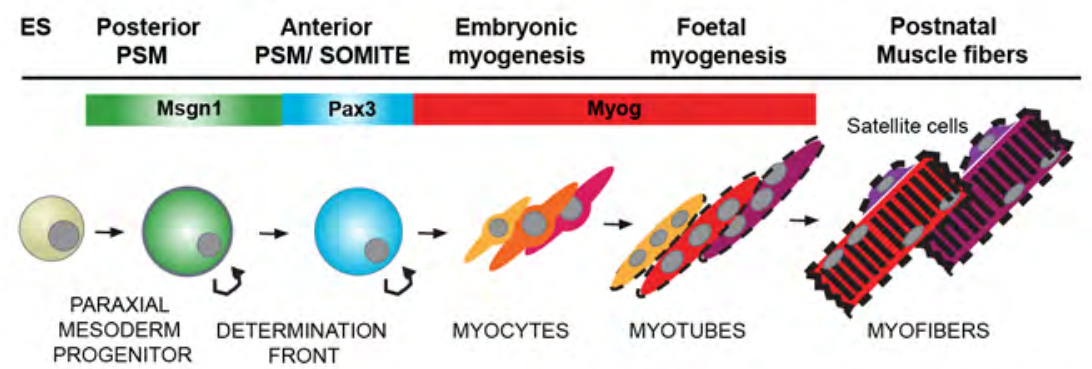

Figure 3 Chal et al 

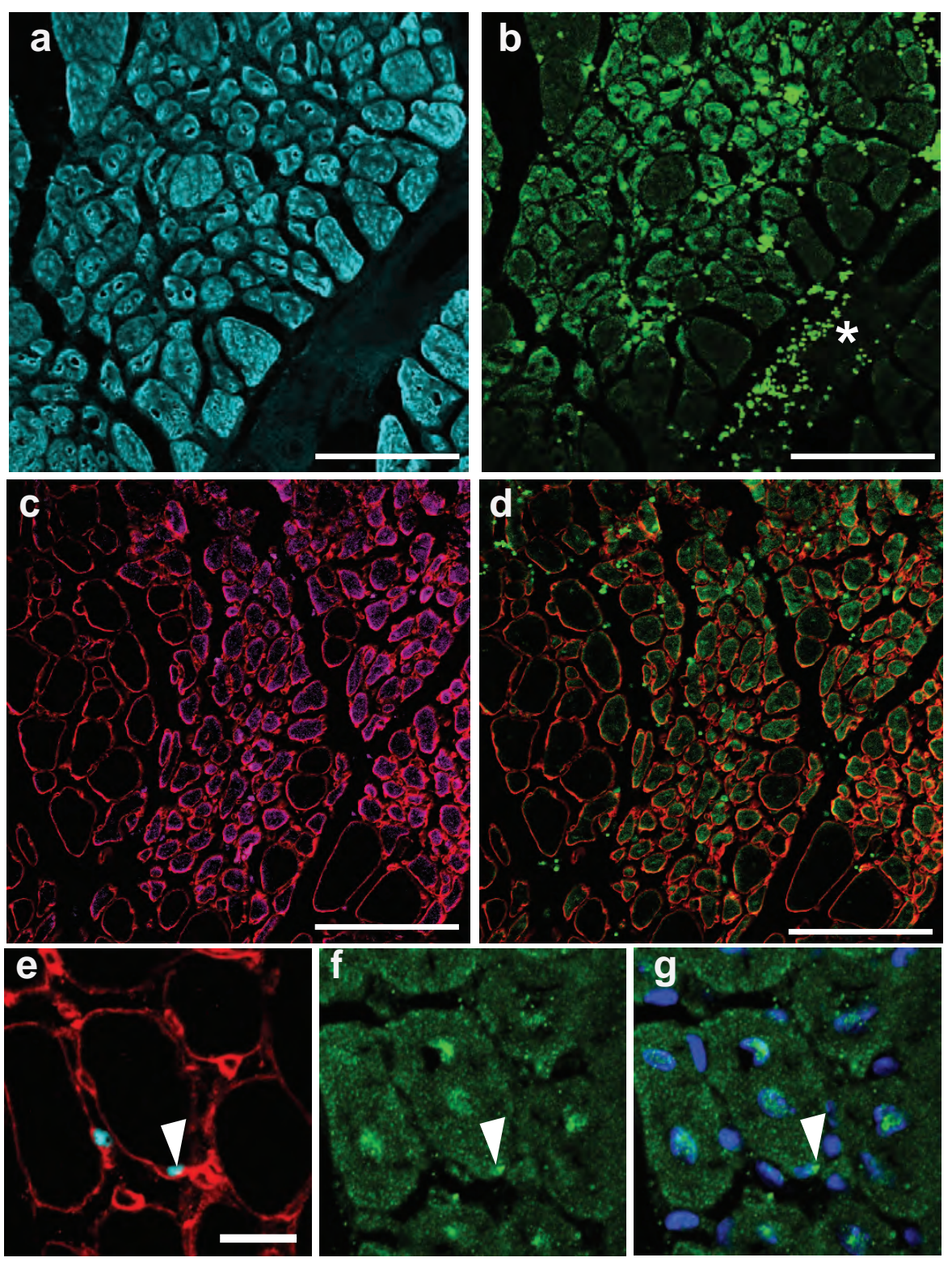

Figure 4 Chal et al 


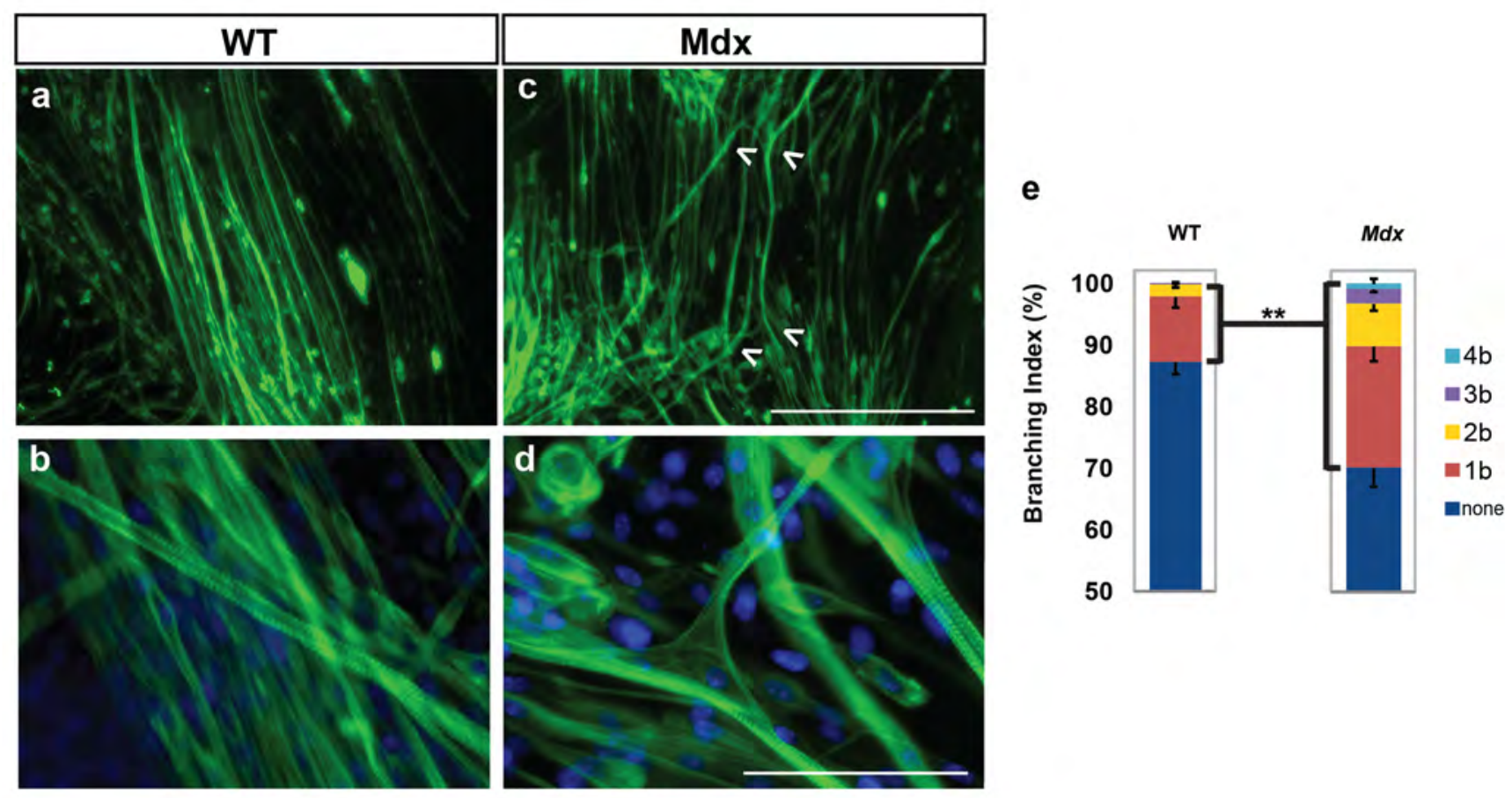

Figure 5 Chal et al 

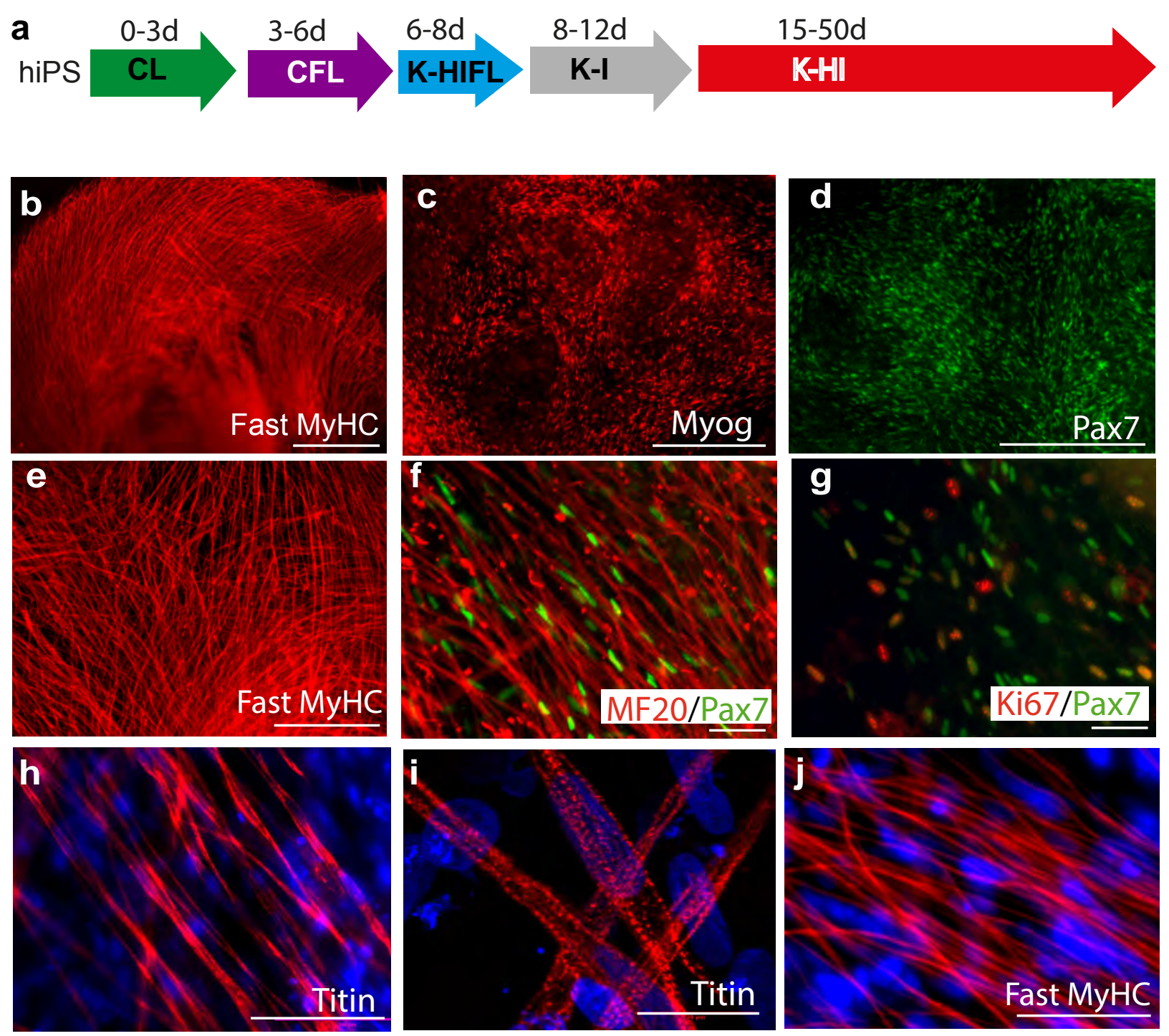

Figure 6 Chal et al 